\title{
Adesão à terapia antirretroviral de pacientes portadores de HIV/Aids com lipodistrofia
}

\author{
Adherence to antiretroviral therapy by HIV/AIDS patients with lipodystrophy \\ Adhesión a la terapia antirretroviral de pacientes VIH / SIDA con lipodistrofia
}

Dalva Francês Costa'; Ana Sofia Resque Gonçalves"; José Ricardo dos Santos Vieirall'; João Farias Guerreirolv

\begin{abstract}
RESUMO
Objetivo: descrever o perfil de adesão à terapia antirretroviral (TARV) de pacientes com Síndrome Lipodistrófica do Vírus da Imunodeficiência Humana (SLHIV), em uma unidade especializada do Estado do Pará, Brasil. Método: estudo qualiquantitativo, envolvendo questionário e prontuários de 124 pacientes, no período de fevereiro e março de 2013, após aprovação por Comitê de Ética. Na análise, a variância $(p<0,05)$ articulou testes estatísticos, com dados apresentados em tabelas. Resultados: as dificuldades de entender e conhecer o esquema terapêutico, o Diabetes Mellitus e as alterações da lipodistrofia foram significantes na interferência da adesão à TARV. O grupo de adesão irregular está em risco para a eficácia do tratamento. Os demais têm a possibilidade de migração de um grupo para outro a qualquer momento. Conclusão: o perfil de adesão à TARV não é definida por dados socioeconômicos. A participação em grupos de adesão deve ser estimulada como fator de reversão do padrão de abandono.

Descritores: HIV; AIDS; lipodistrofia; adesão à medicação.
\end{abstract}

\section{ABSTRACT}

Objective: to profile adherence to antiretroviral therapy (ART) by patients with human immunodeficiency virus lipodystrophy syndrome (HIVLS) at a specialized unit in Pará, Brazil. Method: this quali-quantitative study, involving a questionnaire and medical records of 124 patients, was conducted from February to March 2013, after research ethics approval. In the analysis, statistical tests were related by variance $(p<0.05)$, and data were presented in table form. Results: the difficulties of understanding and knowing the therapeutic regimen, mellitus diabetes and changes in lipodystrophy were significant in the interference of ART adherence. The irregular adherence group is at risk for treatment efficacy. The others have the possibility of migrating from one group to another at any time. Conclusion: the profile of adherence to ART is not defined by socioeconomic data. Participation in membership groups should be encouraged as a factor in reversing the pattern of abandonment.

Descriptors: HIV; SIDA; lipodystrophy; adherence to medication.

\section{RESUMEN}

Objetivo: describir el perfil de adhesión a la terapia antirretroviral (TARV) de pacientes con síndrome lipodistrófico del virus de la inmunodeficiencia humana (SLHIV), en una unidad especializada del Estado de Pará, Brasil. Método: estudio cualiquantitativo involucrando cuestionario e historias clínicas de 124 pacientes, en el período de febrero y marzo de 2013, después de la aprobación por el Comité de Ética. En el análisis, la varianza $(p<0,05)$ articuló pruebas estadísticas, con datos presentados en tablas. Resultados: las dificultades en entender y conocer el esquema terapéutico, la Diabetes Mellitus y las alteraciones de la lipodistrofia fueron significantes en la interferencia de la adhesión a la TARV. El grupo de adhesión irregular está en riesgo para la eficacia del tratamiento. Los demás tienen la posibilidad de migración de un grupo a otro en cualquier momento. Conclusión: el perfil de adhesión a la TARV no está definido por datos socioeconómicos. La participación en grupos de adhesión debe ser estimulada como factor de reversión del patrón de abandono.

Descriptores: VIH; SIDA; lipodistrofia; adhesión a la medicación.

\section{INTRODUÇÃO}

A Síndrome da Imunodeficiência Adquirida (AIDS) tem sido uma das mais importantes pandemias e um dos mais graves problemas de saúde pública mundial. De acordo com a Organização Mundial de Saúde, o agente causador da AIDS, o Vírus da Imunodeficiência Humana vem assumindo papel tão significativo, principalmente, por ter atingido, 34 milhões de pessoas. Destas, 3,3 milhões são crianças menores de 15 anos. Entre os 30,7 milhões de adultos, 14,0 milhões são homens e 16,7 milhões são mulheres, refletindo a contínua feminização da distribuição epidemiológica, invertendo a situação de décadas passadas ${ }^{1,2}$.

Desde 1996, o Brasil adota o acesso universal à terapia antirretroviral (TARV) altamente ativa com gran-

'Enfermeira. Hospital Universitário João de Barros Barreto. Doutoranda em Doenças Tropicais. Universidade Federal do Pará. Brasil. E-mail: dalvafrances@ufpa.br. "Enfermeira. Doutora pela Universidade Federal de Santa Catarina. Professora Associada, Universidade Federal do Pará. Brasil. E-mail: sofiaresque@gmail.com. I'IFarmacêutico. Doutor em Genética e Biologia. Professor Adjunto, Universidade Federal do Pará. Brasil. E-mail: jrvieira@ufpa.br.

${ }^{\text {IV} M e ́ d i c o . ~ D o u t o r ~ e m ~ C i e ̂ n c i a s ~ B i o l o ́ g i c a s . ~ P r o f e s s o r ~ T i t u l a r, ~ U n i v e r s i d a d e ~ F e d e r a l ~ d o ~ P a r a ́ . ~ B r a s i l . ~ E-m a i l: ~ j o a o f g @ u f p a . b r . ~}$ 
des benefícios para as pessoas vivendo com HIV/AIDS. A partir de 2013, o Ministério da Saúde (MS) recomenda o início imediato da TARV em todas as pessoas vivendo com HIV/ AIDS considerando a motivação do paciente ${ }^{3}$. Apesar da eficácia da TARV, as reações adversas aos medicamentos podem ser motivos de diminuição da adesão ao tratamento, o que é um elemento complicador para os programas de saúde pública ${ }^{4}$. É o caso da lipodistrofia, importante efeito colateral, a qual geralmente apresenta um desenvolvimento progressivo dos sinais físicos, com aumento da gravidade, por um período de 18 a 24 meses, estabilizando-se após cerca de dois anos ${ }^{5}$. Os principais fatores de risco para a lipodistrofia são o tempo de infecção pelo HIV, a idade e o tempo da TARV, porém o processo é lento e pode levar anos para ser percebido e incomodar o paciente ${ }^{6}$.

Até o momento não existe tratamento definitivo e curativo para a lipodistrofia, e as diversas estratégias utilizadas no tratamento demonstram um grau variável de sucesso, principalmente relacionados a exercícios físicos, exposição mínima e/ou retardo da TARV, tratamento cirúrgico e orientação nutricional ${ }^{6,7}$. São necessários mais estudos investigativos sobre esta temática, uma vez que as pesquisas sobre adesão em pacientes vivendo com AIDS e com lipodistrofia ainda são insuficientes; assim como, é relevante que se melhorem os registros e acompanhamentos desses pacientes, para se estabelecer uma base de dados mais sólida às novas pesquisas.

Embora a TARV seja imprescindível no tratamento do HIV/AIDS/lipodistrofia e venha dando certo, ainda persistem desequilíbrios na sua adesão. Por isso, objetivou-se, neste trabalho, descrever o perfil de adesão à terapia antirretroviral em pacientes com diagnóstico ou sinais clínicos de lipodistrofia. Independente do momento do tratamento, é preciso analisar suas implicações a fim de contribuir com ações educativas de sensibilização para a adesão à terapia.

\section{REVISÃo DE LITERATURA}

O surgimento dos primeiros casos de AIDS no Brasil, na década de 80 , coincidiu com a instalação de grave crise econômica, social e política no país; provocou intensa mobilização social, que serviu para estimular as primeiras ações políticas em favor da criação do Sistema Único de Saúde, definindo os primeiros passos para se estabelecer respostas governamentais à epidemia da AIDS ${ }^{8,9}$.

O quadro epidemiológico da AIDS apresenta um ciclo de desenvolvimento caracterizado pelo aumento na taxa de incidência e sua manutenção em patamares elevados em todo o mundo; assim, também ocorreu diferentes regiões do Brasil, marcadamente em populações de maior vulnerabilidade ${ }^{10}$. Por se tratar de um problema de saúde pública, cabe aos gestores e profissionais de saúde a responsabilidade de elaborar estratégias eficazes de prevenção e tratamento das pessoas que vivem com a síndrome ${ }^{11}$.
Nessa ótica, compete às academias, através do exercício das atividades docentes, o empenho na formação dos profissionais, buscando fortalecer e intensificar a relação entre o profissional de saúde e o usuário do sistema de saúde, gerando um estado de satisfação a todos os segmentos envolvidos no processo ensino-aprendizagem, garantindo qualidade na prestação de serviços à comunidade ${ }^{12}$.

O relatório anual da Organização Mundial da Saúde (OMS) aponta que, do início da epidemia até junho de 2017, 76 milhões de pessoas foram infectadas pelo HIV e destas, aproximadamente 21 milhões de pessoas tiveram acesso à TARV ${ }^{13}$.

Com a introdução dessa terapia e suas diferentes formas de ação sobre o ciclo do HIV no organismo humano adveio um grande impacto positivo nos indicadores de morbidade, mortalidade e de qualidade de vida dos indivíduos vivendo com HIV/AIDS, o que levou à mudança do status de doença terminal para doença crônica ${ }^{14,15}$. Todavia, apesar da eficácia da TARV, ela é responsável por inúmeros efeitos colaterais importantes, entre eles a lipodistrofia.

A lipodistrofia corresponde a uma redistribuição da gordura corporal e alterações do perfil lipídico e glicêmico. As alterações físicas mais comuns caracterizam-se pelo acúmulo lipídico no abdome, costas, pescoço, nuca, nas regiões submentoniana e pubiana, além de ginecomastia nos homens e aumento de mamas em muIheres. Perda lipídica nos braços, pernas, região glútea e face podendo resultar em proeminência relativa da musculatura e circulação venosa ${ }^{16,17}$.

As alterações corporais que afetam a autoimagem do usuário e comprometem a saúde física e psíquica do paciente, influenciam significativamente no processo de adesão terapêutica ${ }^{18-21}$.

\section{Metodologia}

Trata-se de estudo do tipo descritivo, transversal, não controlado, vinculado a uma abordagem qualiquantitativa. Foi desenvolvido no ambulatório do serviço de assistência da Unidade de Referência Especializada em Doenças Infecciosas e Parasitárias Especiais da Secretaria de Estado de Saúde Pública do Pará (URE/DIP) em Belém, no ano de 2013.

O estudo foi aprovado sob o número 267.224 de 30/04/2013, pelo Comitê de Ética e Pesquisa do Hospital Universitário João de Barros Barreto. Os preceitos éticos-legais foram obedecidos e respeitados no cumprimento dos procedimentos da pesquisa envolvendo seres humanos.

A amostra foi constituída por 124 participantes que compareceram à unidade, no período de fevereiro a março de 2013. A seleção foi pautada nos seguintes critérios: indivíduos com HIV/AIDS, de ambos os sexos, com idade entre 20 e 70 anos (média de 42,4士10,5 anos) 
e que apresentaram sinais clínicos SLHIV ou diagnóstico de lipodistrofia, seguindo as orientações do Ministério da Saúde ${ }^{17}$. Foram excluídos da pesquisa os indivíduos que não se enquadraram nos critérios de diagnóstico estabelecidos e que apresentaram distúrbios metabólicos e/ou lipodistrofia anterior ao diagnóstico de infecção por HIV, mulheres grávidas e lactantes.

A coleta dos dados foi mediante consulta aos prontuários dos pacientes matriculados, que também foram entrevistados. A amostra foi obtida por método não probabilístico. Foi utilizado um questionário para obtenção de dados socioeconômicos (sexo, idade, estado civil, endereço, profissão, renda familiar, escolaridade, tabagismo e hábito regular de ingerir bebidas alcoólicas); com itens para a avaliação da compreensão de fatores importantes para a adesão à TARV (entendimento do esquema terapêutico, efeito colateral da TARV, prática regular de exercícios físicos, comparecimento às consultas com nutricionista, seguimento das orientações alimentares) e sobre sua condição patológica (a lipodistrofia interfere na adesão à TARV, a importância da adesão no tratamento e evolução clínica, a importância da participação no grupo de adesão). Também foi prevista a obtenção dos dados antropométricos, clínicos e laboratoriais para identificar a presença de dislipidemias, obesidade e Diabetes Mellitus (DM).

A partir da informação da suspensão da TARV, foram considerados quatro grupos de adesão/abandono, conforme estabelecido na Nota Técnica № 208/09 - elaborada e publicada pela Secretaria de Vigilância em Saúde (SVS) do Ministério da Saúde (MS): adesão regular (AR): aqueles que mantiveram regularidade; adesão irregular (AI): todos os casos em que o tempo de ausência do paciente para as consultas médicas foi menor que 6 meses, ou ainda, horários de tomada irregular dos medicamentos, esquecimento de doses (1 dia ou mais) e suspensão nos finais de semana; abandonos notificados (AN): todos os casos que foram devidamente notificados ao Ministério da Saúde; e abandono não notificado (ANN): pacientes que descontinuaram a TARV e cujo abandono não foi notificado oficialmente.

A análise de variância de correlação entre as respostas do questionário em relação aos grupos de adesão/abandono foi realizada por meio do Teste de Kruskal-Wallis, para indicar o quanto as diferenças entre as respostas em cada grupo foram discriminantes para a diferenciação dos grupos. Para comparar variáveis presentes em pacientes com adesão regular, em relação aos demais grupos, foi realizada análise discriminante, determinação de discriminante canônica, os testes $T$ de Student, Qui Quadrado, G e Exato de Fisher, de acordo com a variável, utilizando o programa Bioestat5.0 ${ }^{\circledR}$. Para todas as análises foi considerado como grau de significância de $p<0,05$. As informações obtidas foram armazenadas em um banco de dados plotados em planilhas eletrônicas do MS Office Excel (versão 2007) e apresentados em tabelas.

\section{RESULTADOS}

Participaram da pesquisa 124 pacientes portadores de HIV/AIDS com lipodistrofia, ocorrendo a presença majoritária de homens ( $n=85 / 67 \%$ ) e minoritária de mulheres ( $n=39 / 32 \%$ ), em uma relação de 2,2 homens para cada mulher; 68 ( 55\%) participantes eram solteiros, 69 (55,70\%) residiam na cidade de Belém, 82 (66\%) com renda familiar de até um salário-mínimo, 97 ( 78\%) pacientes cursaram o Ensino Fundamental completo. Houve predomínio de pacientes não etilistas, 89 (72\%), e não tabagistas, 110 (89\%). Constatou-se que 26 (21\%) participantes sabiam conceituar lipodistrofia, 12 (46,2\%) aprenderam o conceito na própria unidade, $8(30,8 \%)$ na internet e $6(23,0 \%)$ em outras fontes.

Evidenciou-se que $75(60,48 \%)$, pacientes atribuíram algum tipo de reação adversa à TARV, entre os quais $17(22,70 \%)$ relataram que a lipodistrofia era o efeito que mais os incomodava. Somente no prontuário de 78 (100\%) pacientes havia informações sobre dislipidemias, dos quais $44(56,40 \%)$ apresentavam registros dessas alterações laboratoriais.

Quanto à adesão ao tratamento, 100 (80,60\%) pacientes confirmaram saber sua importância. Entre estes, $44 \%$ apontaram o aumento da sobrevida e a melhora da saúde como os principais benefícios alcançados com o uso correto da TARV ( $22 \%$ para cada variável). A melhora da qualidade de vida, como o maior benefício obtido, foi apontada por 18 (18\%) pacientes .

Em relação à atividade física, mesmo sendo uma das recomendações de controle e tratamento das alterações provocadas pela lipodistrofia, 111 (89,52\%) pacientes não a realizavam; do mesmo modo, 112 (90,30\%) não compareciam ao serviço para controle e orientação dietética; destes, 74 (66,10\%) alegaram desinteresse ou acharam desnecessário, 21 (18,70\%) alegaram desconhecimento ou falta de tempo; 7 (6,3\%), por não terem sido encaminhados, e $10(8,9 \%)$ alegaram outros motivos.

Tiveram dificuldade em entender o esquema terapêutico, 38 pacientes (30,70\%); destes, 21 (16,90\%) por causa do horário e 17 (17,80\%) pelo ajuste das doses com os horários. Fizeram a suspensão do medicamento por conta própria em algum momento, 89 pacientes $(71,80 \%)$.

O sentimento de revolta foi relatado por 19 $(21,34 \%)$ pacientes como motivo do abandono, 17 $(19,10 \%)$ citaram a presença da lipodistrofia; e outros motivos, que variaram desde a negação da doença até viagens e uso de drogas ilícitas, conforme Tabela 1.

Quanto ao grupo de adesão, 106 (85,47\%) pacientes não compareceram às reuniões, 78 (62,90\%) alegaram desconhecer o significado e a existência desse grupo. A qualidade de vida não foi afetada com a lipodistrofia para $71(57,26 \%)$ dos pesquisados . Vale ressaltar que 50 (40,32\%) alegaram que a lipodistrofia causava várias interferências, que iam desde constrangimento até discriminação, de acordo com a Tabela 2. 
As respostas, entre os pacientes nos grupos de adesão/abandono, foram variadas.. Somente quatro respostas mostraram diferenças significativas $(p<$ $0,05)$ discriminando cada grupo de adesão/abandono e foram consideradas cruciais para definir o perfil de abandono ou adesão irregular ou regular à TARV. Tais respostas estão distribuídas por quatro grupos, segundo a Tabela 3.

TABELA 1: Motivo alegado pelos pacientes HIV/AIDS, com diagnóstico ou sinais sugestivos de lipodistrofia, quanto ao padrão de regularidade no uso da TARV (abandono ou adesão irregular). Belém, Pará, Brasil, 2013.

\begin{tabular}{|c|c|c|c|c|c|c|c|c|}
\hline \multirow[t]{2}{*}{ Motivo alegado } & \multicolumn{6}{|c|}{ Padrão de regularidade no uso da TARV } & \multicolumn{2}{|c|}{ Total } \\
\hline & AN & $\%$ & ANN & $\%$ & Al & $\%$ & $\mathbf{n}$ & $\%$ \\
\hline Revolta & 7 & 29,20 & 5 & 31,25 & 7 & 14,2 & 19 & 21,34 \\
\hline Lipodistrofia & 2 & 8,20 & 3 & 18,75 & 12 & 24,4 & 17 & 19,10 \\
\hline Negação da doença & 4 & 16,60 & 3 & 18,75 & 7 & 14,2 & 14 & 15,73 \\
\hline Viagens & 6 & 25,00 & 1 & 6,25 & 7 & 14,2 & 14 & 15,73 \\
\hline Alcoolismo & 1 & 4,20 & 1 & 6,25 & 3 & 6,5 & 5 & 5,62 \\
\hline Esquecimento & 1 & 4,20 & - & - & 4 & 8,5 & 5 & 5,62 \\
\hline Religiosos & 1 & 4,20 & 2 & 12,50 & 2 & 4,00 & 5 & 5,62 \\
\hline Depressão & - & - & 1 & 6,25 & 2 & 4,00 & 3 & 3,37 \\
\hline Dificuldades para deslocamento & - & - & - & - & 2 & 4,00 & 2 & 2,25 \\
\hline Descrédito no tratamento & 1 & 4,20 & - & - & 1 & 2,00 & 2 & 2,25 \\
\hline Datas comemorativas & - & - & - & - & 1 & 2,00 & 1 & 1,12 \\
\hline Disfagia & - & - & - & - & 1 & 2,00 & 1 & 1,12 \\
\hline Uso de drogas ilícitas & 1 & 4,20 & - & - & - & - & 1 & 1,12 \\
\hline Total & 24 & 26,97 & 16 & 17,98 & 49 & 55,05 & 89 & 100 \\
\hline
\end{tabular}

AN = abandono notificado $\mathrm{ANN}=$ abandono não notificado $\mathrm{Al}=$ adesão irregular

Sinal convencional utilizado igual a zero não resultante de arredondamento.

TABELA 2: Interferência das alterações provocadas pela lipodistrofia nas atividades da vida diária, em pacientes HIV/ AIDS, com diagnóstico ou sinais sugestivos de lipodistrofia. Belém, Pará, Brasil, 2013.

\begin{tabular}{lccccc}
\hline $\begin{array}{l}\text { Interferências das alterações provocadas pela } \\
\text { lipodistrofia nas atividades da vida diária }\end{array}$ & \multicolumn{2}{c}{ Padrão de regularidade no uso da TARV } & \multicolumn{2}{c}{ Total } \\
\hline Não interferem & 15 & ANN & Al & AR & 18 \\
Constrangimento & 6 & 6 & 31 & 10 & 28 \\
Baixa autoestima & 1 & 3 & 5 & 5 & 14 \\
Prejudicou as relações afetivas & - & - & 2 & 1 & 3 \\
Prejudicou as relações profissionais & 1 & - & - & - & 1 \\
Dificulta as atividades & - & - & 1 & 1 & 2 \\
Discriminação & - & - & 2 & - & 2 \\
Não sabe & 1 & - & 1 & - & 2 \\
Não respondeu & - & - & 1 & - & 1 \\
Total & $\mathbf{2 4}$ & $\mathbf{1 6}$ & $\mathbf{4 9}$ & $\mathbf{3 5}$ & $\mathbf{1 2 4}$ \\
\hline
\end{tabular}

AN = abandono notificado; $A N N$ = abandono não notificado; $\mathrm{Al}=$ adesão irregular; $\mathrm{AR}$ = adesão regular. Sinal convencional utilizado dado numérico igual a zero não resultante de arredondamento.

TABELA 3: Respostas sobre os itens considerados discriminantes para os tipos de adesão e abandono à terapia antirretroviral em pacientes de HIV/AIDS com lipodistrofia. Belém, Pará, Brasil, 2013.

\begin{tabular}{|c|c|c|c|c|c|c|c|c|}
\hline \multirow[t]{2}{*}{ Grupos } & \multicolumn{2}{|c|}{$\begin{array}{c}\text { Dificuldade em } \\
\text { entender o esquema } \\
\text { terapêutico }\end{array}$} & \multicolumn{2}{|c|}{$\begin{array}{l}\text { Diabetes mellitus } \\
\text { (não/sim) }^{(*)}\end{array}$} & \multicolumn{2}{|c|}{$\begin{array}{l}\text { Não compreende que } \\
\text { lipodistrofia pode } \\
\text { interferir na adesão à } \\
\text { TARV }\end{array}$} & \multicolumn{2}{|c|}{$\begin{array}{l}\text { Compreende a } \\
\text { importância da } \\
\text { adesão à TARV }\end{array}$} \\
\hline & $f$ & $\%$ & $f$ & $\%$ & $f$ & $\%$ & f & $\%$ \\
\hline AN & 17 & 13,7 & $13 /-$ & $16,5 /-$ & 20 & 16,1 & 15 & 12,1 \\
\hline ANN & 9 & 7,3 & 8/- & 10,1/- & 14 & 11,3 & 12 & 9,7 \\
\hline $\mathrm{Al}$ & 29 & 23,4 & $32 / 1$ & $40,5 / 1,3$ & 48 & 38,7 & 40 & 32,3 \\
\hline AR & 31 & 25,0 & $20 / 5$ & $25,3 / 6,3$ & 35 & 28,2 & 33 & 26,6 \\
\hline Total & 86 & 69,4 & $73 / 6$ & $92,4 / 7,6$ & 117 & 94,4 & 100 & 80,7 \\
\hline Valor pt & \multicolumn{2}{|c|}{0,019} & \multicolumn{2}{|c|}{0,041} & \multicolumn{2}{|c|}{0,016} & \multicolumn{2}{|c|}{0,021} \\
\hline
\end{tabular}

${ }^{(*)}$ Dados calculados a partir de 79 prontuários; † Teste de Kruskal-Wallis.

AN = abandono notificado; ANN = abandono não notificado; $\mathrm{Al}=$ adesão irregular; $\mathrm{AR}$ = adesão regular; TARV = terapia antirretroviral. Sinal convencional utilizado igual a zero não resultante de arredondamento. 
Considerando o padrão de regularidade à TARV, 24 ( 19,40\%) casos foram classificados como abandono notificado (AN), 16 (12,90\%) como abandono não notificado (ANN), 49 (39,50\%) como adesão irregular (AI) e 35 (28,20\%) como adesão regular (AR). No grupo ANN, o de maior índice, $50 \%$ foram classificados corretamente e atenderam às características inerentes ao ANN. Dos casos de $\mathrm{Al}$, somente $22 \%$ foram classificados corretamente e $46 \%$ dos casos de AR, foram classificados corretamente.

Para se avaliar o padrão de agrupamento dos quatro grupos de adesão/abandono foi realizada a análise de função discriminadora canônica, discriminando a dispersão dos grupos entre si e os valores centroides de cada grupo representando a média dos resultados. Os centroides dos quatros grupos surgem muito próximos entre si, correndo uma dispersão intensa e significativa dos elementos que compõem os quatro grupos, onde os pacientes tiveram os seus perfis distribuídos, de acordo com a característica de cada grupo. A variação ideal seria que os centroides estivessem dispersos entre si e os componentes (indivíduos), o mais agregado possível ao centroide representante de seu grupo.

\section{DIsCUSSÃo}

Os resultados revelaram que os dados socioeconômicos (gênero, idade, estado civil, preocedência profissão, renda familiar, escolaridade, tabagismo e hábito regular de ingerir bebidas alcoólicas) não foram significativos no processo de adesão, tampouco discriminantes para o abandono da terapia antirretroviral, em associação similar a vários estudos ${ }^{5,20,21}$.

O mesmo foi observado em relação a variáveis que possuem relação direta com a lipodistrofia (reação adversa que provoca maior desconforto, presença de dislipidemia e prática de exercícios físicos; acompanhamento nutricional e medidas de controle alimentar; o conhecimento sobre lipodistrofia, grupo de adesão e participação de reuniões), que não apresentaram significância estatística quando relacionadas à adesão e ao abandono à TARV de maneira contrária a outros estudos ${ }^{6}$.

Apesar da predominância da resposta negativa à prática de exercícios físicos pelos pacientes, no presente estudo não houve associação estatística significativa entre a prática de exercícios físicos e o processo de adesão/abandono. O exercício físico regular pode ser benéfico, tanto para as alterações metabólicas, como provavelmente também na redistribuição de gorduras central e intramiocelular e deve ser considerada para todos os pacientes infectados pelo HIV6.

O sedentarismo que, associado às dislipidemias, não apresentou relação significativa com o padrão de adesão/abandono. Este resultado, a priori, não parece justificável, considerando que as atividades físicas têm um importante papel na redução de peso, diminuição de massa corpórea gorda, aumento de massa corpórea magra, redução da pressão sanguínea e aumento da sensibilidade à insulina ${ }^{6}$. Esta falta de conexão é um indicativo de que os indivíduos não relacionaram esses fatores com o aparecimento da lipodistrofia e/ou com a complicação do seu quadro clínico, fatow observável pelo alto índice de não comparecimento às reuniões dos grupos de adesão e às consultas com nutricionista ${ }^{7}$.

Quatro variáveis apresentaram associação estatística significativa: dificuldades de entender o esquema terapêutico; presença de DM; compreensão que a lipodistrofia pode interferir na adesão à TARV; e conhecimento sobre a importância da adesão à TARV. Esses resultados são similares a outros estudos que indicam horários e número de doses, a quantidade, o sabor e o tamanho dos comprimidos ${ }^{7}$.

A dificuldade em entender o esquema terapêutico é um dado importante, à medida que reflete a qualidade do serviço ofertado pela unidade de saúde responsável pelo atendimento e, sobretudo, implica imediata revisão da forma como estão sendo passadas as informações sobre a tomada dos medicamentos. O sabor, o tamanho, a forma de armazenamento, a quantidade de comprimidos e outros fatores podem conflitar com as rotinas e o estilo de vida, levando o paciente a apresentar certa dificuldade para entender o esquema terapêutico ${ }^{7}$. Resultados de pesquisas divergentes ${ }^{22}$ reformam que questões relacionadas aos conhecimentos adquiridos na unidade de saúde pelos pacientes, alcançaram um nível satisfatório (78,7\% a 95,7\%), indicando que havia uma adequada satisfatória quanto à importância da adesão ao tratamento ${ }^{22}$.

A presença de diabetes foi uma variável discriminante para a adesão. Porém, dos 124 investigados, somente em 79 prontuários havia a confirmação clínico-laboratorial dos pacientes diabéticos e não diabéticos, o que pode trazer um viés à análise, visualizado em um valor $p$ limiar ao valor de corte 0,05 . Porém, observou-se que dentre os seis diabéticos da amostra, cinco possuíam adesão regular e somente um indivíduo diabético teve adesão irregular à TARV. A ingestão de um número maior de drogas/dia, típica dos pacientes diabéticos, pode melhorar a adesão em virtude de uma rotina de administrar medicamentos em horários fixos ser mais assimilada. Resultados discordantes são mostrados em estudos onde, pelo contrário, a ingestão conjunta de vários comprimidos relacionados a comorbidades prejudica a adesão à TARV22.

Com relação à compreensão de que lipodistrofia pode interferir na adesão à TARV, é um fator discriminante dos grupos de adesão, uma vez que a maioria dos indivíduos, que compreendia a importância da lipodistrofia, correspondia aos grupos de adesão regular $(38,7 \%)$ e adesão irregular (28,2\%). Da mesma forma, o conhecimento sobre a importância da adesão à TARV, foi confirmado por 26,6\% (AR) e 32,3\% (AI). Contudo, quando se particularizou a questão relacionada aos efeitos colaterais, observou-se que a lipodistrofia foi 
discriminante no processo de abandono e adesão ao tratamento com antirretrovirais $(p=0,016)$; vale destacar uma vez que as mudanças corporais prejudicam a autoimagem do paciente, provocando uma redução da qualidade de vida nesses indivíduos, e, consequentemente, determinam menor adesão ao tratamento, incorrendo muitas vezes em falha terapêutica ${ }^{23}$.

Mesmo nos casos de adesão regular, quando foi questionado sobre os impactos psicológicos e os efeitos sobre a realização das atividades da vida diária, apesar de todos apresentarem lipodistrofia, apenas 10 (47,6\%) deles relataram impacto profundo na vida diária. Este fato, todavia, não deflagrou nenhuma interrupção de tratamento por conta própria, mas dificultaram em muito a aceitação dessas modificações do corpo, as quais foram assim identificadas: percepção negativa da autoimagem; evitar contatos sociais; redução da autoconfiança; mudanças em estados de humor; sentimento de frustração diante da ausência de resultados satisfatórios e percepção negativa da autoestima.

A magnitude do problema pode ser constatada na fala dos próprios pacientes, quando cogitam abandonar a TARV, em busca de um corpo mais harmônico, em virtude das mudanças corporais que são percebidas por outras pessoas e que os leva a evitar sair de casa, por temerem o reconhecimento pelas pessoas da estigmatizante cara da AIDS ${ }^{24,25}$.

Observou-se que não há um comportamento padrão de agrupamento dos grupos de adesão regular à TARV em relação aqueles que abandonam o tratamento; assim, não há garantias de que alguém com adesão regular ao tratamento não venha a abandoná-lo, bem como aqueles que abandonam, possam voltar a ter regularidade no tratamento.

\section{CONCLUSÃo}

Os dados socioeconômicos não definem o perfil de adesão/abandono à TARV, porém, a maioria de indivíduos com adesão irregular essa terapia revela que está em risco para a eficácia do tratamento. Dados como a suspensão temporária do tratamento nos finais de semana e datas festivas, para fazer uso de bebida alcoólica, devem ser acompanhados como um importante fator para a adesão irregular, que pode se caracterizar pela suspensão temporária, que varia de um a 72 meses, o que mostra que é muito frequente a suspensão temporária, mas podendo atingir períodos muito longos de suspensão.

O acompanhamento psicológico mostra-se importante nesse grupo de indivíduos, uma vez que a revolta, e a presença da lipodistrofia foram apontadas, pela maioria dos pacientes, como as principais determinantes da irregularidade do tratamento.

É notória a necessidade de melhorar o desempenho do grupo junto aos pacientes, por se tratar de uma atividade de suma importância para a adesão dos pacientes ao tratamento, além de ser uma orientação do
Ministério da Saúde. Entender o esquema terapêutico é determinante para definir o processo de adesão, pois o paciente consegue administrar as doses e horários do seu tratamento com mais facilidade. É necessário que os profissionais responsáveis em orientar o uso do esquema terapêutico se certifiquem do entendimento do paciente com relação às drogas prescritas, como horário, doses, e até mesmo, o aspecto visual de cada medicamento, para facilitar a compreensão e minimizar os erros de ingestão, qualificando a adesão.

Entre as limitações do estudo, ressaltam-se a incompreensão do problema por parte dos pacientes, falta de apoio dos profissionais e a falta de registros nos prontuários. Esses fatos dificultaram a avaliação consistente dos achados e limitam a generalização dos resultados.

Novos estudos poderão mostrar ações implementadas para a melhoria da compreensão do problema, por parte do paciente e da equipe de apoio, revisando ao padrão de regularidade à TARV, bem como comparar outras estratégias adotadas por diferentes centros de tratamento para aprimorar a adesão nessa terapia.

\section{REFERÊNCIAS}

1. Ministério da Saúde (Br). Aids no Brasil: epidemia concentrada e estabilizada em populações de maior vulnerabilidade. Boletim Epidemiológico. 2012; 43(1): 8-10.

2. Ministério da Saúde (Br). DST e Aids. Boletim Epidemiológico. 2014; 3(1): 80.

3. Dye C. After 2015: infectious diseases in a new era of health and development. Phil. Trans. R. Soc. B 369: 20130426. 2014; 369(1645): 20130426. doi: 10.1098/rstb. 2013.0426.

4. Prosperi MC, Fabbiani M, Fanti I, Zaccarelli M, Colafigli M, Mondi A et al. Predictors of first-line antiretroviral therapy discontinuation dueto drug-related adverse events in HIV-infected patients: a retrospective cohort study. BMC. Infect. Dis. 2012; 12(12):296. 5. Araújo MNR, Mendes PR, Silveira APP, Seidl EMF. Lipodistrofia e preconceito na nova cara da AIDS: diálogo com a bioética de intervenção. Revista Bioética, 2011. [citado em 15 mar 2017]; 19(2): 441-56. Disponível em: http://revistabioetica.cfm.org.br/index.php. 6. Signorini DJHP, Monteiro MCM, Andrade MFC, Signorini DH, Eyer-Silva WA. O que devemos saber sobre síndrome metabólica e lipodistrofia na AIDS? Rev. Assoc. Med. Bras. 2012; 58(1): 70-5. 7. Galvão MTG, Gouveia AS, Fiúza MLT, Costa E. Reflexões de portadores de HIV/AIDS acerca da terapia antirretroviral. Rev. Rene. 2011; 12(esp): 966-72.

8. Maliska ICA, Padilha MICS, Andrade SR. AIDS e as primeiras respostas voltadas para a epidemia: contribuições dos profissionais de saúde. Rev. enferm. UERJ. 2015; 23(1): 15-20.

9. Leadebal ODCP, Medeiros LB, Lins KSM, Chaves RB, Monroe AA, Nogueira JA. Cuidado às pessoas vivendo com AIDS: enfoque nas ações de educação em saúde. Rev. enferm. UERJ. 2017; 25: e9524. 10. Ministério da Saúde $(\mathrm{Br})$. Departamento de DST, Aids e Hepatites Virais. Política brasileira de enfrentamento da AIDS: resultados, avanços e perspectivas. [Internet]. 2012 [citado em:18 ago 2017]. Disponível em: https://www.academia.edu/7891408/ Politica_brasileira_enfrentamento_aids_2012_2_

11. Silva RAR, Costa RHS, Nelson ARC, Du9arte FHS, Prado NCC, Rodrigues EHF. Fatores preditivos dos diagnósticos de enfermagem em pessoas vivendo com a síndrome da imunodeficiência adquirida. Rev. latinoam. enferm. (Online). 2016 [citado em 15 jun 2017]; 24: e2712. Disponível em: http://www.eerp.usp.br/rlae. 12. Gonçalves ASR, Pires DEP. O trabalho de docentes universitários da saúde: situações geradoras de prazer e sofrimento. Rev. 
enferm. UERJ. 2015; 23(2): 266-71.

13. Organização Mundial de Saúde. Relatório UNAIDS 2017. [internet]. 2017. [citado em 10 nov 2017]. Disponível em: https:// unaids.org.br/tag/relatório-unaids-2017/

14. Giralt M, Domingo P, Villarroya F. Adipose tissue biologyand hiv-infections. Best Pract. Res. Clin. Endocrinol. Metab. 2011; 25(3): 487-99.

15. Feleke Y, Fekade D, Mezegebu Y. Prevalence of highly active antiretroviral therapy associated metabolic abnormalities and lipodystrophy in HIV infected patients. Ethiop. Med. J. 2012; 50(3): 221-30.

16. Lartey M, Asante-Quashie A, Essel A, Kenu E, Ganu V, Neequaye A. Adverse drug reactions to antiretroviral therapy during the early art period at a tertiary hospital in Ghana. Pan. Afr. Med. J. 2014; 7(18):25.

17. Ministério da Saúde (Br). Secretaria de Vigilância em Saúde. Programa Nacional de DST e Aids. Síndrome lipodistrófica em HIV. Brasília (DF): MS; 2011 [citado em 18 nov 2017]. Disponível em: http://docplayer.com.br/1993038-Ministerio-da-saude-sindrome-lipodistrofica-em-hiv.html.

18.Saber Viver Comunicação. Entenda melhor a lipodistrofia. Edições Especiais. [Internet], 2015. [citado em 18 nov 2017] Disponível em: http://saberviver.org.br/publicacoes/entenda-melhor-a-lipodistrofia/

19. Finkelstein JL, Gala P, Rochford R, Glesby MJ, Mehta S. HIV/
AIDS and lipodystrophy: implications for clinical management in resource-limited settings. J. Int. AIDS Soc. 2015; 18(1):19033. doi: 10.7448/IAS.18.1.19033. eCollection 2015.

20.Zambrini H. Estudo de dados clínicos e laboratoriais de pacientes com AIDS e lipodistrofia atendidos no programa de lipodistrofia do Hospital Heliópolis [dissertação de mestrado]. São Paulo: Universidade de São Paulo; 2011.

21.Schilkowsky LB, Portela MC, SÁ MC. Fatores associados ao abandono de acompanhamento ambulatorial em um serviço de assistência especializada em HIV/AIDS na cidade do Rio de Janeiro. Rev. Bras. Epidemiol. 2011; 14(2): 187-97.

22. Wilson G, Wollf M. A decade of antiretroviral therapy: a profile of patients with 10 years of highly effective triple therapy. Rev. Chilena de Infectol. 2012; 29(3): 337-43.

23. Souza Junior PRB, Szwarcwald CL, Castilho EA. Self-ratedhealthby HIV-infected individual sunder going antiretroviral therapy in Brazil. Cad. Saúde Pública (Online). 2011 [Internet]; 27(supl.1). [cited 2015 Mar 12]. Available from: http://dx.doi.org/10.1590/S0102.

24. Fiuza MLT, Lopes EM, Alexandre HO, Dantas PB, Galvão MTG, Pinheiro AKB. Adesão ao tratamento antirretroviral: assistência integral baseada no modelo de atenção às condições crônicas. Esc. Anna Nery. Rev. Enferm. 2013; 17 (4): 740-8.

25. Aragonés C, Sánchez L, Campos JR, Pérez J. Antiretroviral therapy adherence in persons with HIV/AIDS in Cuba. MEDICC Rev. 2011; 13(2): 17-23. 